Document downloaded from:

http://hdl.handle.net/10251/70969

This paper must be cited as:

Barrachina Villalba, J.; Juan-Carlos Cano; Tavares De Araujo Cesariny Calafate, CM.; Manzoni ., P. (2013). V2X-d: a Vehicular Density Estimation System that combines V2V and V2I Communications. IEEE IEEE Catalog Number CFP1314F-USB. 1-6.

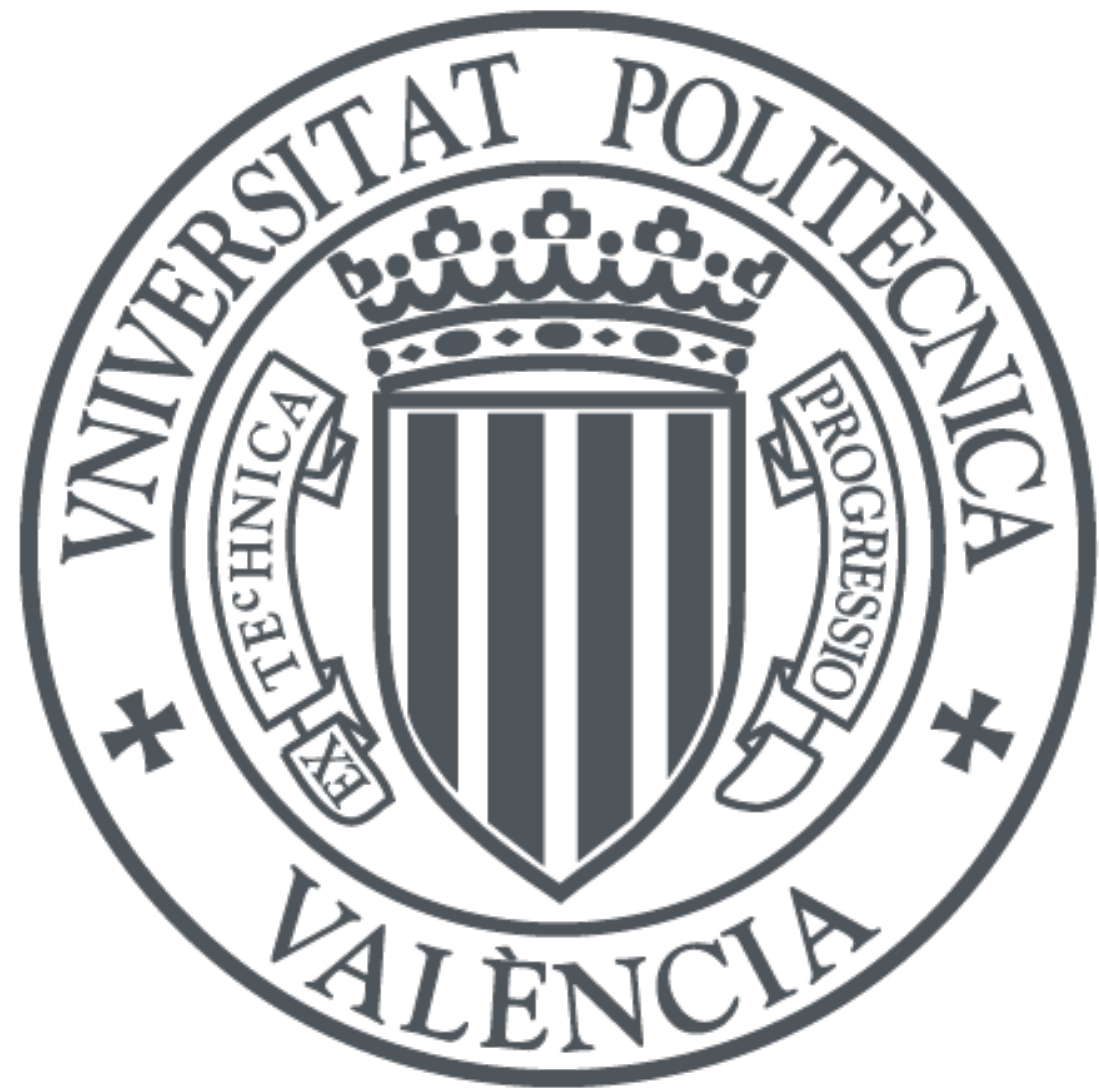

The final publication is available at

http://dx.doi.org/10.1109/WD.2013.6686518

Copyright IEEE

Additional Information

(C) 2013 IEEE. Personal use of this material is permitted. Permission from IEEE must be obtained for all other uses, in any current or future media, including reprinting/republishing this material for advertising or promotional purposes, creating new collective works, for resale or redistribution to servers or lists, or reuse of any copyrighted component of this work in other works. 


\section{V2X-d: a Vehicular Density Estimation System that combines V2V and V2I Communications}

\author{
Javier Barrachina, Julio A. Sanguesa, Manuel Fogue, \\ Piedad Garrido, Francisco J. Martinez \\ Computer Science and System Engineering Department \\ University of Zaragoza, Spain \\ Email: \{barrachina, jsanguesa, mfogue, \\ piedad, f.martinez\}@unizar.es
}

\author{
Juan-Carlos Cano, Carlos T. Calafate, Pietro Manzoni \\ Computer Engineering Department \\ Universitat Politècnica de València, Spain \\ Email: \{jucano, calafate, pmanzoni\}@disca.upv.es
}

\begin{abstract}
Road traffic is experiencing a drastic increase, and vehicular traffic congestion is becoming a major problem, especially in metropolitan environments throughout the world. Additionally, in modern Intelligent Transportation Systems (ITS) communications, the high amount of information that can be generated and processed by vehicles will significantly increase message redundancy, channel contention, and message collisions, thus reducing the efficiency of message dissemination processes. In this work, we present a V2X architecture to estimate traffic density on the road that relies on the advantages of combining V2V and V2I communications. Our proposal uses both the number of beacons received per vehicle $(\mathrm{V} 2 \mathrm{~V})$ and per RSU (V2I), as well as the roadmap topology features to estimate the vehicle density. By using our approach, modern Intelligent Transportation Systems will be able to reduce traffic congestion and also to adopt more efficient message dissemination protocols.

Index Terms-Vehicular Networks, VANETs, vehicle density estimation, Road Side Unit, V2V, V2I, V2X.
\end{abstract}

\section{INTRODUCTION}

Intelligent Transportation Systems (ITS) involve both traffic management and vehicle communication capabilities. Specifically, modern ITS services can efficiently manage information on the road, being able to offer drivers a variety of added services such as safe, efficient, and smart driving.

Regarding transportation, road traffic is experiencing a drastic increase, and vehicular traffic congestion is becoming a major problem, especially in metropolitan environments throughout the world. In particular, traffic congestion: (i) reduces the efficiency of the transportation infrastructure, (ii) increases travel time, fuel consumption, and air pollution, and (iii) leads to increased user frustration and fatigue [1]. Traditionally, vehicle density has been one of the main metrics used for assessing road traffic conditions. A high vehicle density usually indicates congested traffic; however, the density of vehicles in a city highly varies depending on the area and the time during the day. Thus, knowing the density of a vehicular environment is important since it allows applying traffic congestion countermeasures focused on improving traffic flow, reducing contamination, and increasing drivers' comfort.

Regarding communications, Vehicular Networks (VNs) are wireless communication networks that support cooperative driving among communicating vehicles on the road.
VNs involve vehicle-to-vehicle (V2V) [2] and vehicle-toinfrastructure (V2I) [3] communications, and have received a remarkable attention in recent years. The specific characteristics of vehicular networks favor the development of attractive and challenging services and applications. Though traffic safety has been the primary motive for the development of these networks, VNs also facilitate applications for managing the traffic flow, monitoring road conditions, environmental protection, and mobile infotainment [4]. Most of these applications could behave more efficiently if the protocols involved become aware of the density of vehicles at any given time, being able to adapt their behavior according to this factor. Hence, knowing the traffic density in vehicular scenarios is of great importance since it promotes using the wireless channel more efficiently, thereby improving ITS wireless-based services.

Currently, most of the vehicle density estimation approaches are designed to use very specific infrastructure-based traffic information systems, which require the deployment of vehicle detection devices such as inductive loop detectors, or traffic surveillance cameras [5], [6]. However, these approaches are limited since they can only be aware of traffic density in a priori selected areas (i.e., the streets and junctions in which these devices are already located), making it difficult to estimate the vehicular density along a whole city. In addition, some of these approaches are not able to perform accurate estimations in real time (e.g., using cameras involves intensive image processing and analysis). The use of V2I communications can address the aforementioned problems [7].

Other authors, such as Stanica et al. [8] and Sanguesa et al. [9], propose estimating the traffic density by using V2V communications. According to these approaches, each vehicle is able to estimate the number of nearby vehicles by taking into account beacon information. However, such proposals are only capable of obtaining information about density in their neighborhood, while being unable to infer traffic density for the whole scenario. Hence, vehicles are unable to determine the best route to avoid traffic jams.

Since using V2V or V2I communication approaches separately presents some advantages, but also some drawbacks, in this work we present a V2X architecture to estimate traffic 


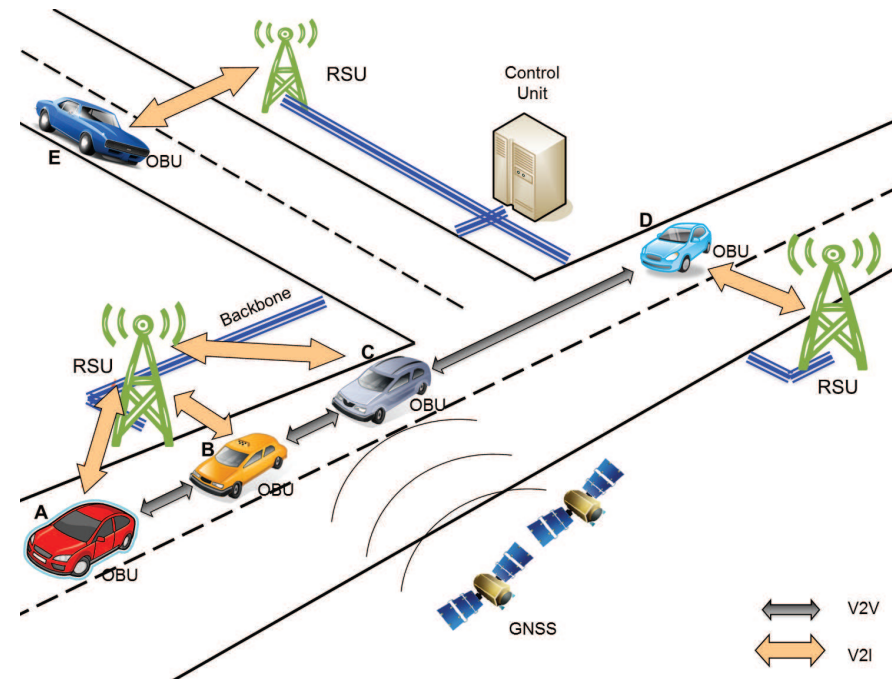

Fig. 1. Architecture of the V2X-based vehicle density estimation system.

density on the road that combines V2V and V2I communications. Unlike previous proposals, our approach maximizes the advantages of V2V (accuracy, microscopic approach, etc.) with the advantages of V2I (global information, macroscopic approach, etc.). Our proposal uses both the number of beacons received per vehicle (V2V) and per RSU (V2I), as well as the roadmap topology features, to estimate the vehicle density. By using our proposal, modern Intelligent Transportation Systems will be able to reduce traffic congestion and also to adopt more efficient message dissemination protocols.

This paper is organized as follows: In Section II we present the V2X-d architecture. Section III presents the simulation environment. Section IV details our V2X real-time density estimation proposal. In Section V we compare our V2X-d proposal with other density estimation approaches. Section VI introduces the most important applications of the V2X-d architecture. Finally, Section VII concludes this paper.

\section{V2X-D ARCHITECTURE FOR ACCURATE DENSITY ESTIMATION IN VEHICULAR ENVIRONMENTS}

Figure 1 presents an overview of our proposed architecture. As shown, each vehicle incorporates an On-Board Unit (OBU) responsible of the wireless communication, hence providing the necessary network interfaces to implement the density estimation mechanism using the data exchanged by the vehicles on the road, i.e., V2V communication. In addition, the OBU must have access to positioning devices corresponding to Global Navigation Satellite Systems (GNSS) such as the Global Positioning System (GPS) [10], or the future Galileo [11] system developed by the European Union. This information may be critical to determine areas of interest for the estimation, or to predict the vehicles' movements to improve future estimations.

The development of the V2I part of the vehicular communication requires the installation of additional infrastructure nodes, called Road Side Units (RSUs), that provide support to the vehicles and are able to offer additional services. RSU deployment also allows communication between different areas of the map if necessary, by connecting to a common backbone [12].

Using this proposed architecture, the deficiencies of the different systems used, i.e., V2V and V2I, can be addressed through the combination of both technologies. Vehicles can achieve a global awareness of the network around them, whereas the RSUs can improve their information about traffic at microscopic levels through the local information obtained from the vehicles. This opens a wide variety of possibilities to obtain an accurate vehicle density estimation, overcoming blind spots or sparsely connected areas.

In order to achieve an accurate density estimation, we first need to develop systems able to determine the density of vehicles by using V2V and V2I communications. Moreover, this system should provide adequate results independently of the scenario in which the communication takes place. That is, the estimation mechanism should adapt to the features of the road topology.

\section{Simulation EnVironment}

All the simulations performed in this work were done using the ns-2 simulator, where the PHY and MAC layers have been modified to closely follow the IEEE $802.11 \mathrm{p}$ standard $^{1}$, which defines enhancements to 802.11 required to support ITS applications. We assume that all the nodes are equipped with an IEEE 802.11p interface tuned at the frequency of $5.9 \mathrm{GHz}$ for both V2V and V2I communications.

In terms of the physical layer, the data rate used for packet broadcasting was $6 \mathrm{Mbit} / \mathrm{s}$, as this is the maximum rate for broadcasting in $802.11 \mathrm{p}$. The MAC layer was also extended to include four different priorities for channel access. Therefore, application messages are categorized into four different Access Categories (ACs), where AC0 has the lowest and AC3 the highest priority.

Regarding V2I communications, we used the Uniform Mesh deployment policy [12] to deploy RSUs in the maps. This approach consists on distributing RSUs uniformly on the map. The advantage of this deployment policy is that it achieves a more uniform coverage area since the distance between RSUs is the same, preventing RSUs to be deployed too closely, or too sparsely. As for the mobility model, it has been obtained with CityMob for Roadmaps (C4R) [13], a mobility generator able to import maps directly from OpenStreetMap [14], and generate ns-2 compatible traces. Table I shows the parameters used for the simulations.

Table II shows the main features of each map for the cities under study: the number of streets, the number of junctions, the average segment size, and the number of lanes per street. According to the results obtained in previous work [9], we consider that the parameters that better correlate with the complexity of the roadmap are the number of streets and the

\footnotetext{
${ }^{1}$ All these improvements and modifications are available at http://www.grc.upv.es/software/
} 
TABLE I

PARAMETERS USED FOR THE SIMULATIONS

\begin{tabular}{|l|c|}
\hline Parameter & Value \\
\hline & New York, Minnesota, Madrid, \\
roadmaps & San Francisco, Los Angeles, \\
& Amsterdam, Sydney, Liverpool, \\
Valencia, Rio de Janeiro, and Rome \\
roadmap size & $2000 \mathrm{~m} \times 2000 \mathrm{~m}$ \\
number of vehicles & {$[100,200,300 \ldots 1000]$} \\
warning messages priority & $A C 3$ \\
beacon priority & $A C 1$ \\
interval between messages & $1 \mathrm{~second}$ \\
number of RSUs & 9 \\
RSU deployment policy & Uniform Mesh [12] \\
MAC/PHY & $802.11 \mathrm{p}$ \\
radio propagation model & $\mathrm{RAV}[15]$ \\
mobility model & Krauss [16] \\
channel bandwidth & $6 \mathrm{Mbps}$ \\
max. transmission range & $400 \mathrm{~m}$ \\
\hline
\end{tabular}

TABLE II

MAP FEATURES

\begin{tabular}{|c|c|c|c|c|c|}
\hline Map & Streets & Junctions & $\begin{array}{c}\text { Avg. segment } \\
\text { size (m.) }\end{array}$ & $\begin{array}{c}\text { Lanes/ } \\
\text { street }\end{array}$ & SJ Ratio \\
\hline New York & 257 & 500 & 45.8853 & 1.5730 & 0.5140 \\
\hline Minnesota & 459 & 591 & 102.0652 & 1.0144 & 0.7766 \\
\hline Madrid & 628 & 715 & 83.0820 & 1.2696 & 0.8783 \\
\hline San Francisco & 725 & 818 & 72.7065 & 1.1749 & 0.8863 \\
\hline Los Angeles & 283 & 306 & 408.2493 & 1.1448 & 0.9379 \\
\hline Amsterdam & 1494 & 1449 & 44.8973 & 1.1145 & 1.0311 \\
\hline Sydney & 872 & 814 & 72.1813 & 1.2014 & 1.0713 \\
\hline Liverpool & 1758 & 1502 & 49.9620 & 1.2295 & 1.1704 \\
\hline Valencia & 2829 & 2233 & 33.3653 & 1.0854 & 1.2669 \\
\hline Rio de Janeiro & 542 & 401 & 167.9126 & 1.1135 & 1.3516 \\
\hline Rome & 1655 & 1193 & 45.8853 & 1.0590 & 1.3873 \\
\hline
\end{tabular}

number of junctions. Hence, we added a column labeled as SJ Ratio, which represents the result of dividing the number of streets between the number of junctions. As shown, the first 5 cities (New York, Minnesota, Madrid, San Francisco, and Los Angeles) present an SJ ratio smaller than 1, which indicates that they have a simple topology, while the rest of the cities (Amsterdam, Sydney, Liverpool, Valencia, Rio de Janeiro, and Rome) present a higher SJ value, which indicates that they have a complex topology. According to this, complex maps like Valencia obtain worse results in terms of vehicular communications than simple maps such as Madrid, where the wireless signal is able to reach a higher number of vehicles.

\section{OuR V2X Density Estimation System}

In this section, we proceed to obtain both $\mathrm{V} 2 \mathrm{~V}$ and $\mathrm{V} 2 \mathrm{I}-$ based functions to estimate traffic density, with the minimum possible error. We consider necessary to obtain both estimation functions, since the information provided by each one can be applied in different kinds of applications. For instance, applications with the goal of reducing broadcast storm problems and avoiding traffic overhead in vehicular networks only need to know the traffic density within the neighborhood of each vehicle. However, centralized systems that aim at avoiding traffic jams need information about the entire area being managed to be capable of rerouting vehicles to areas with less traffic density.
TABLE III

V2V FUNCTION COEFFICIENTS

\begin{tabular}{|c|c|}
\hline Coeff. & Value \\
\hline a & $-1.1138191190298828 \mathrm{E}+03$ \\
\hline b & $-1.0800433554686800 \mathrm{E}+01$ \\
\hline c & $3.1832185406821718 \mathrm{E}+03$ \\
\hline d & $-4.0336415134812398 \mathrm{E}-01$ \\
\hline f & $-3.0203454502011946 \mathrm{E}+03$ \\
\hline g & $2.8542014049626700 \mathrm{E}-03$ \\
\hline h & $9.5199929660347175 \mathrm{E}+02$ \\
\hline i & $3.5319225007012626 \mathrm{E}+01$ \\
\hline$j$ & $1.6230525995036607 \mathrm{E}-01$ \\
\hline k & $-1.6615888771467137 \mathrm{E}+01$ \\
\hline
\end{tabular}

TABLE IV

V2V DENSITY ESTIMATION ERROR

\begin{tabular}{|c|c|c|}
\hline Error & Absolute & Relative \\
\hline Minimum & $-2.61203 \mathrm{E}+01$ & $-2.28480 \mathrm{E}-01$ \\
\hline Maximum & $2.16953 \mathrm{E}+01$ & $5.71311 \mathrm{E}-01$ \\
\hline Mean & $-3.17620 \mathrm{E}-10$ & $1.02334 \mathrm{E}-02$ \\
\hline Std. Error of Mean & $1.36030 \mathrm{E}+00$ & $1.71408 \mathrm{E}-02$ \\
\hline Median & $1.69890 \mathrm{E}-01$ & $-1.35912 \mathrm{E}-03$ \\
\hline
\end{tabular}

\section{A. V2V-based density estimation}

In order to propose a V2V-based method able to accurately estimate the density of vehicles, we made a total of 4000 experiments. These experiments involved the simulation of controlled scenarios (i.e., scenarios where the actual density is known). According to the results obtained, we propose a density estimation function capable of estimating the vehicular density in every urban environment, at any instant of time.

To determine the best approach, we have tested some different functions (exponential, logarithmic, etc.). With this goal in mind, we performed a regression analysis by using ZunZun [17] that allowed us to find the polynomial equation offering the best fit to the data obtained through simulation. Equation 1 shows the density estimation function, which is able to estimate the number of vehicles per $\mathrm{km}^{2}$ in urban scenarios, according to the number of beacons received, and the SJ ratio (i.e., streets/junctions) of the selected roadmap.

$$
\begin{array}{r}
f(x, y)=a+b x+c y+d x^{2}+f y^{2}+g x^{3}+ \\
+h y^{3}+i x y+j x^{2} y+k x y^{2}
\end{array}
$$

In this equation, $x$ is the number of beacons received by each vehicle, and $y$ is the SJ ratio obtained from the roadmap. The values of the polynomial coefficients $(a, b, c, d, f, g, h, i, j$, and $k)$ are listed in Table III.

To determine the accuracy of our proposal, we proceed to measure the estimated error. Table IV shows the different types of errors calculated when comparing our density estimation function with the values actually obtained. Note that the average relative error is of only $1.02 \%$.

\section{B. V2I-based density estimation}

Similarly to the V2V-based vehicle density estimation approach, we proceed to obtain a function to estimate vehicle density. To this end, we performed a regression analysis that allowed us to find an equation offering the best fit to the 
TABLE V

V2I FUNCTION COEFFICIENTS

\begin{tabular}{|c|c|}
\hline Coeff. & Value \\
\hline a & $2.3037584774238823 \mathrm{E}+02$ \\
\hline b & $1.9069648769466475 \mathrm{E}+01$ \\
\hline c & $-4.2946130569906342 \mathrm{E}+02$ \\
\hline d & $3.1880957532509228 \mathrm{E}+01$ \\
\hline f & $1.8795302200929001 \mathrm{E}+02$ \\
\hline g & $-6.8125878716641097 \mathrm{E}+01$ \\
\hline
\end{tabular}

TABLE VI

V2I DENSITY ESTIMATION ERROR

\begin{tabular}{|c|c|c|}
\hline Error & Absolute & Relative \\
\hline Minimum & $-5.39939 \mathrm{E}+01$ & $-1.22576 \mathrm{E}+00$ \\
\hline Maximum & $4.83735 \mathrm{E}+01$ & $1.69779 \mathrm{E}+00$ \\
\hline Mean & $2.84849 \mathrm{E}-13$ & $3.04107 \mathrm{E}-02$ \\
\hline Std. Error of Mean & $2.42242 \mathrm{E}+00$ & $3.54373 \mathrm{E}-02$ \\
\hline Median & $2.37153 \mathrm{E}-01$ & $1.58332 \mathrm{E}-03$ \\
\hline
\end{tabular}

data obtained through simulation. We select Equation 2 as the V2I-based density estimation function, since it obtained the smallest relative error.

$f(x, y)=a+b \cdot \ln (x)+\frac{c}{y}+d \cdot \ln (x)^{2}+\frac{f}{y^{2}}+\frac{g \cdot \ln (x)}{y}$

In this equation, $x$ is the average number of beacons received by each RSU, and $y$ is the $\mathrm{SJ}$ ratio obtained from the roadmap. The values of the coefficients $(a, b, c, d, f$, and $g$ ) are listed in Table V.

To determine the accuracy of our proposal, we proceed to measure the estimated error. Table VI shows the different errors when comparing our density estimation function with the values actually obtained through simulation. Note that the average relative error is of only 3.04\%. As expected, the V2Ibased vehicle density estimation approach presents a higher estimation error than the V2V-based approach, but it is almost negligible for the majority of ITS applications. In addition, the use of V2I can enrich and complete the estimation obtained by the V2V approach.

Moreover, by using our system, we are able to estimate the vehicular density in more specific areas. For example, we can identify areas where the traffic is more congested (i.e., areas where the RSUs receive a higher percentage of beacons). According to this, an automatic traffic control system could take advantage from V2I communication capabilities to adapt the vehicles' routes in order to redirect vehicles traveling in more congested areas to those areas where the RSUs receive a lower number of messages (i.e., less congested), thus avoiding traffic jams.

\section{COMPARISON WITH OTHER DENSITY ESTIMATION APPROACHES}

As mentioned above, other vehicular density estimation proposals rely on the use of infrastructure elements such as surveillance cameras, inductive loop detectors, or ambient microphones to estimate the vehicle density (e.g., [1], [5], and [6]). The use of V2I communications has also been studied (e.g., [3], [7]). Those approaches require to deploy RSUs, although they can provide attractive and value-added services
TABLE VII

V2V BEACONS-ONLY FUNCTIONS COEFFICIENTS

\begin{tabular}{|c|c|c|c|c|}
\hline Coefficient & Quadratic & Cubic & Quartic & Preece-Baines \\
\hline a & $1.82943 \mathrm{E}+01$ & $2.27684 \mathrm{E}+01$ & $3.90472 \mathrm{E}+01$ & $1.90872 \mathrm{E}+02$ \\
\hline b & $4.13673 \mathrm{E}+00$ & $3.29413 \mathrm{E}+00$ & $-1.38471 \mathrm{E}+00$ & $1.63278 \mathrm{E}+02$ \\
\hline c & $-2.15091 \mathrm{E}-02$ & $7.02894 \mathrm{E}-03$ & $2.97587 \mathrm{E}-01$ & $2.56730 \mathrm{E}-02$ \\
\hline d & - & $-2.55584 \mathrm{E}-04$ & $-6.47130 \mathrm{E}-03$ & $4.40558 \mathrm{E}+01$ \\
\hline f & - & - & $4.27413 \mathrm{E}-05$ & $6.86664 \mathrm{E}-01$ \\
\hline
\end{tabular}

TABLE VIII

V2I BEACONS-ONLY FUNCTIONS COEFFICIENTS

\begin{tabular}{|c|c|c|}
\hline Coefficient & Quintic Polynomial & Quadratic Logarithmic \\
\hline a & $1.35094 \mathrm{E}+01$ & $-3.34403 \mathrm{E}+02$ \\
\hline b & $2.34872 \mathrm{E}-02$ & $2.02972 \mathrm{E}+02$ \\
\hline c & $8.79406 \mathrm{E}-01$ & $-1.82578 \mathrm{E}+01$ \\
\hline d & $-3.74059 \mathrm{E}-02$ & $1.49093 \mathrm{E}+00$ \\
\hline f & $5.62751 \mathrm{E}-04$ & - \\
\hline g & $-2.82498 \mathrm{E}-06$ & - \\
\hline
\end{tabular}

when compared to the former. On the contrary, the proposals based on $\mathrm{V} 2 \mathrm{~V}$ communications do not require the deployment of any infrastructure nodes, but, unlike our proposal, they usually take into account just the number of beacons received (e.g., [8], [18]), while omitting any data related to the map topology where the vehicles are located.

\section{A. Performance comparison of our V2V approach against} other V2V-based density estimation approaches

In order to assess the importance of the topology, we compared our proposal with a beacon-based approach, where the vehicular density is estimated by only using the number of beacons received. To make a fair comparison, we followed the same methodology in both approaches.

We tested four different density estimation functions which are solely based on the number of beacons received, trying to obtain the lowest value for the Sum of Squared Errors (SSE). Specifically, we have tested three different polynomial functions (i.e., quadratic, cubic, and quartic), and a nonpolynomial function (based on the Preece-Baines Growth function). Equations 3-6 show these functions, and Table VII shows their coefficients.

$$
\begin{gathered}
f(x)=a+b x+c x^{2} \\
f(x)=a+b x+c x^{2}+d x^{3} \\
f(x)=a+b x+c x^{2}+d x^{3}+f x^{4} \\
f(x)=\frac{a-2 \cdot(a-b)}{(\exp (c \cdot(x-d))+\exp (f \cdot(x-d)))}
\end{gathered}
$$

Table IX shows the sum of squared errors for each of the functions tested. As shown, our V2V-d function yields more accurate results, presenting the lower sum of squared absolute error $(6.33215 \mathrm{E}+03$, two orders of magnitude lower than the rest), and it has only an average error of 8.90 vehicles, whereas the rest of the functions that only account for the number of beacons show an average error ranging from 40.50 to 41.57 vehicles, depending on the selected function. 
TABLE IX

COMPARISON BETWEEN OUR V2X-D AND THE BEACONS-ONLY DENSITY ESTIMATION APPROACHES

\begin{tabular}{|c|c|c|}
\hline Fitted function & Sum of Squared Errors & Avg. vehicles error \\
\hline Beacons-only Quadratic & $1.38234 \mathrm{E}+05$ & 41.57 \\
\hline Beacons-only Cubic & $1.37994 \mathrm{E}+05$ & 41.53 \\
\hline Beacons-only Quartic & $1.36094 \mathrm{E}+05$ & 41.25 \\
\hline $\begin{array}{c}\text { Beacons-only } \\
\text { Preece-Baines Growth }\end{array}$ & $1.31231 \mathrm{E}+05$ & 40.50 \\
\hline V2V-d & $6.33215 \mathrm{E}+03$ & 8.90 \\
\hline \hline Quintic Polynomial & $1.89925 \mathrm{E}+05$ & 45.94 \\
\hline Quadratic Logarithmic & $2.01613 \mathrm{E}+05$ & 47.33 \\
\hline V2I-d & $4.70035 \mathrm{E}+04$ & 22.85 \\
\hline
\end{tabular}

\section{B. Performance comparison of our V2I approach against} other V2I-based density estimation approaches

Similarly to the V2V-based vehicle density estimation approach, we tested several density estimation functions which are solely based on the number of beacons received, trying to obtain the lowest value of SSE. In particular, we obtained the quintic polynomial function shown in Equation 7, and the quadratic logarithmic function shown in Equation 8.

$$
\begin{gathered}
f(x)=a+b x+c x^{2}+d x^{3}+f x^{4}+g x^{5} \\
f(x)=a+b \cdot \ln (d x)+c \cdot \ln (d x)^{2}
\end{gathered}
$$

The results presented in Table IX confirm that our V2I-d function provides more accurate results than the other V2I beacons-only density estimation functions, presenting a low value for the Sum of Squared Errors (i.e., 4.70035E+04), whereas the beacons-based functions present a Sum of Squared Errors value of 1.89925E+05 (for the polynomial) and 2.01613E+05 (for the logarithmic), i.e., one order of magnitude higher than our proposal. Specifically, its average error is of only 22.85 vehicles, whereas the rest of the functions that only account for the number of beacons have an error of 45.94 and 47.33 vehicles, respectively. As shown, the $\mathrm{V} 2 \mathrm{~V}$ density estimation is more accurate, but, as stated before, this approach also presents some drawbacks since it cannot be used in traffic jam avoidance systems. Hence, we consider that a method that combines both V2V and V2I communications is necessary.

\section{Qualitative comparison of our V2X-d approach against other density estimation approaches}

To conclude this section, Table X presents a summary of the different vehicle density estimation methods focusing on their main characteristics. As shown, there is no density estimation approach that can fulfill all the desired capabilities needed by traffic control authorities and modern ITS services, with the exception of the proposed V2X-d approach.

Traditional infrastructure-based methods, such as surveillance cameras or ambient microphones, are highly affected by the environmental conditions (bad light, adverse weather, etc.). Although inductive loop detectors can overcome these problems, similarly to previous approaches, their area of potential density estimation is relatively small (i.e., the streets and junctions where the data acquisition devices are located), they are not fault tolerant, and, obviously, they cannot be used to mitigate broadcast storms since they do not involve wireless communications.

V2I density estimation approaches exhibit good traffic congestion control capabilities, but they are limited in other important functions such as broadcast mitigation features, or fault tolerance (e.g., whenever an RSU stops working or malfunctions, the density estimation inside its target area will become unavailable). As for V2V approaches, they can overcome all these problems, but they cannot be used to provide optimal routes to vehicles; notice that vehicle density estimation is always limited since each vehicle obtains its own neighbor density estimation. Our V2X-d architecture brings together the benefits of both approaches (i.e., V2V and V2I), providing great possibilities to authorities, transport agencies, and drivers in terms of traffic control, travel time reduction, vehicle emissions control, as well as better and faster wireless communications.

\section{V2X-D APPLICATIONS}

Our V2X-d architecture can support a wide variety of useful ITS applications. In this section we present the most remarkable ones that we envision.

- Reducing broadcast storm. Broadcasting messages blindly may lead to packet collisions and channel congestion and contention, which drastically reduces the performance of message delivery schemes [19]. Using the density estimated by means of $\mathrm{V} 2 \mathrm{~V}$ communication, the vehicles can determine when they are competing with a high number of vehicles for the channel, and thus limiting the transmission of non-critical information to avoid channel saturation.

- Avoiding traffic jams, reducing $\mathrm{CO} 2$ emissions. The global knowledge provided by V2I communication and the information exchanged by the RSUs allow detecting areas with a high traffic density, thus more prone to traffic jams. Vehicles can be informed about these areas, and thus modify their routes to reach their destination sooner.

- Reducing the emergency services arrival time to the location where an accident has taken place, routing these emergency vehicles through streets with a small vehicle density, or rerouting the rest of vehicles to improve the emergency vehicles' path time.

- Collecting historical data about more frequently traveled zones, with the goal of increasing road maintenance in areas with more traffic, or allowing authorities to better plan future actions.

- Adjusting vehicle density estimation when vehicles or RSUs are no able to collect all the needed information separately. During RSU deployment, some areas may fail to have RSU coverage, creating a blind spot that will prevent achieving an accurate global estimation. This could lead to discrepancies between the estimated density and the real density of vehicles located in the 
TABLE X

QuALITATIVE COMPARISON OF THE DifFERENT DENSITY ESTIMATION APPROACHES

\begin{tabular}{|c|c|c|c|c|c|c|}
\hline Feature & Cameras & $\begin{array}{c}\text { Loop } \\
\text { detectors }\end{array}$ & Microphones & V2I & V2V & V2X-d \\
\hline $24 / 7$ availability & $\bar{x}$ & $\checkmark$ & $\sqrt{ }$ & $\checkmark$ & $\checkmark$ & $\checkmark$ \\
\hline All-sound conditions & $\sqrt{ }$ & $\boldsymbol{V}$ & $\bar{x}$ & $\boldsymbol{V}$ & $\sqrt{ }$ & $\sqrt{ }$ \\
\hline Different light conditions & $\bar{x}$ & $\checkmark$ & $\bar{v}$ & $\bar{v}$ & $\boldsymbol{V}$ & $\boldsymbol{V}$ \\
\hline All-Weather conditions & $\bar{x}$ & $\boldsymbol{V}$ & $\boldsymbol{x}$ & $\boldsymbol{V}$ & $\sqrt{ }$ & $\sqrt{ }$ \\
\hline Wide coverage & $\overline{\boldsymbol{x}}$ & $\bar{x}$ & $\bar{x}$ & $\sqrt{ }$ & $\bar{x}$ & $\sqrt{ }$ \\
\hline Real-time estimation & $\bar{x}$ & $\boldsymbol{V}$ & $\boldsymbol{V}$ & $\boldsymbol{V}$ & 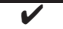 & 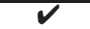 \\
\hline Traffic jams avoidance & $\sqrt{ }$ & $\sqrt{ }$ & $\sqrt{ }$ & $\sqrt{ }$ & $\overline{\boldsymbol{x}}$ & $\sqrt{ }$ \\
\hline Broadcast storms mitigation & $\bar{x}$ & $\bar{*}$ & $\bar{x}$ & $\bar{x}$ & $\sqrt{ }$ & $\checkmark$ \\
\hline Fault tolerant & $\bar{x}$ & $\overline{\boldsymbol{x}}$ & $\overline{\boldsymbol{x}}$ & $\bar{x}$ & $\sqrt{ }$ & $\sqrt{ }$ \\
\hline
\end{tabular}

area. However, using the data collected by means of V2V communication, the RSUs can complete the missing information and adjust the estimation. This strategy can also be used to overcome failures in the system, such as damaged infrastructure elements, backbone errors, and so on. Having both V2V and V2I communication available helps at obtaining more insight about the traffic characteristics, increasing the fault tolerance of the system.

\section{CONCLUSIONS}

This paper proposes V2X-d, an architecture that allows estimating vehicle density in urban environments at any given time by combining both V2V and V2I communications. Our proposal allows improving proactive traffic congestion mitigation mechanisms to better redistribute vehicles' routes, while adapting them to the specific traffic conditions. Additionally, it allows implementing more efficient and adaptive information dissemination schemes. Unlike existing proposals, our V2X$\mathrm{d}$ vehicular density estimation architecture accounts not only for the number of beacons received, but also for the map topology in the region where the vehicles are located. We demonstrate how our approach is able to accurately predict the vehicular density. Moreover, our proposal can solve the problems associated to existing approaches, (e.g., those caused by bad light conditions, adverse weather, etc.).

\section{ACKNOWLEDGMENTS}

This work was partially supported by the Ministerio de Ciencia e Innovación, Spain, under Grant TIN2011-27543C03-01, by the Fundación Universitaria Antonio Gargallo and the Obra Social de Ibercaja, under Grant 2013/B010, as well as the Government of Aragón and the European Social Fund (T91 Research Group).

\section{REFERENCES}

[1] V. Tyagi, S. Kalyanaraman, and R. Krishnapuram, "Vehicular traffic density state estimation based on cumulative road acoustics," IEEE Transactions on Intelligent Transportation Systems, vol. 13, no. 3, pp. 1156-1166, Sept. 2012.

[2] M. Ng and S. T. Waller, "A static network level model for the information propagation in vehicular ad hoc networks," Transportation Research Part C: Emerging Technologies, vol. 18, no. 3, pp. 393-407, 2010, 11th IFAC Symposium: The Role of Control.

[3] F. Soldo, R. Lo Cigno, and M. Gerla, "Cooperative synchronous broadcasting in infrastructure-to-vehicles networks," in Fifth Annиal Conference on Wireless on Demand Network Systems and Services (WONS), Jan. 2008, pp. 125-132.
[4] F. Malandrino, C. Casetti, C.-F. Chiasserini, and M. Fiore, "Optimal content downloading in vehicular networks," IEEE Transactions on Mobile Computing, vol. 12, no. 7, pp. 1377-1391, 2013.

[5] B. Coifman and H. Lee, "Analytical tools for loop detectors and traffic monitoring systems," in IEEE Intelligent Transportation Systems Conference (ITSC), 2007, pp. 1086-1091.

[6] E. Tan and J. Chen, "Vehicular traffic density estimation via statistical methods with automated state learning," in IEEE Conference on Advanced Video and Signal Based Surveillance (AVSS), Sept. 2007, pp. $164-169$.

[7] J. Barrachina, P. Garrido, M. Fogue, F. J. Martinez, J.-C. Cano, C. Calafate, and P. Manzoni, "I-VDE: A Novel Approach to Estimate Vehicular Density by Using Vehicular Networks," in Ad-hoc, Mobile, and Wireless Network, ser. Lecture Notes in Computer Science, J. Cichon, M. Gebala, and M. Klonowski, Eds. Springer Berlin Heidelberg, 2013, vol. 7960, pp. 63-74.

[8] R. Stanica, E. Chaput, and A. Beylot, "Local density estimation for contention window adaptation in vehicular networks," in IEEE 22nd International Symposium on Personal Indoor and Mobile Radio Communications (PIMRC), Sept. 2011, pp. 730-734.

[9] J. A. Sanguesa, M. Fogue, P. Garrido, F. J. Martinez, J.-C. Cano, C. T. Calafate, and P. Manzoni, "An infrastructureless approach to estimate vehicular density in urban environments," Sensors, vol. 13, pp. 23992406, 2013.

[10] E. Lassiter, "Navstar global positioning system: A satellite based microwave navigation system," in IEEE-MTT-S International Microwave Symposium, 1975, pp. 334-334.

[11] R. Prasad and M. Ruggieri, Applied satellite navigation using GPS, GALILEO, and augmentation systems. Artech House, 2005.

[12] J. Barrachina, P. Garrido, M. Fogue, F. J. Martinez, J.-C. Cano, C. T. Calafate, and P. Manzoni, "Road side unit deployment: A density-based approach," IEEE Intelligent Transportation Systems Magazine, vol. 5, no. 3, pp. 30-39, 2013.

[13] M. Fogue, P. Garrido, F. J. Martinez, J.-C. Cano, C. T. Calafate, and P. Manzoni, "A Realistic Simulation Framework for Vehicular Networks," in 5th International ICST Conference on Simulation Tools and Techniques (SIMUTools 2012), Desenzano, Italy, March 2012, pp. $37-46$.

[14] OpenStreetMap, "Collaborative project to create a free editable map of the world," 2012, available at http://www.openstreetmap.org.

[15] F. J. Martinez, M. Fogue, C. K. Toh, J.-C. Cano, C. T. Calafate, and P. Manzoni, "Computer simulations of VANETs using realistic city topologies," Wireless Personal Communications, vol. 69, no. 2, pp. 639663, 2013.

[16] S. Krauss, P. Wagner, and C. Gawron, "Metastable states in a microscopic model of traffic flow," Physical Review E, vol. 55, no. 5, pp. 5597-5602, 1997.

[17] ZunZun, "Online Curve Fitting and Surface Fitting Web Site," 2013, available at http://www.zunzun.com.

[18] N. Maslekar, M. Boussedjra, J. Mouzna, and H. Labiod, "A stable clustering algorithm for efficiency applications in VANETs," in 7th International Wireless Communications and Mobile Computing Conference (IWCMC), July 2011, pp. 1188-1193.

[19] Y.-C. Tseng, S.-Y. Ni, Y.-S. Chen, and J.-P. Sheu, "The broadcast storm problem in a mobile ad hoc network," Wireless Networks, vol. 8, pp. 153-167, 2002. 